\section{ORIGINAL RESEARCH}

I. Yuki

I. Kan

H.V. Vinters

R.H. Kim

A. Golshan

F.A. Vinuela

J.W. Sayre

Y. Murayama

F. Vinuela

\title{
The Impact of Thromboemboli Histology on the Performance of a Mechanical Thrombectomy Device
}

\begin{abstract}
BACKGROUND AND PURPOSE: Recently we published a novel method of thrombus preparation for use in a swine model for evaluation of thrombectomy designs. The clot (fibrin rich clot) is characterized by its similarity in histologic characteristics to the thromboemboli recovered from stroke patients. The purpose of this latest study was to evaluate if the performance of a mechanical thrombectomy device was affected by the histologic characteristics of thromboembolus. Erythrocyte rich clot, which was created using exogenous thrombin, and the novel experimental clot with abundance of fibrin/cellular component were used for comparison. The Merci clot retriever was used for the evaluation and the angiographic outcomes were analyzed.
\end{abstract}

MATERIALS AND METHODS: Two histologically different types of experimental clot, a conventionally used thrombin-induced clot (erythrocyte-rich clot) and a novel experimental clot that is similar in histologic characteristics to the thromboemboli recovered from patients with stroke (fibrin-rich clot), were prepared. Eight extracranial arteries in swine were occluded with erythrocyte-rich clot (group A), and 8 were occluded with fibrin-rich clot (group B), and MT by using the Merci clot retriever device was performed. Angiographic results in each group were evaluated.

\begin{abstract}
RESULTS: A total of 48 attempts at MT were made. The average number of attempts to achieve TIMI grade II or III recanalization was 2.75 times in group $A$ and 4.5 times in group $B(P<.001)$, respectively. The mean time to achieve recanalization was 15.5 minutes in group $A$ and 81.5 minutes in group $B(P<$ .01). Every vessel in group A showed recanalization (100\%), whereas only 3 of 8 samples (37.5\%) achieved recanalization in group B.
\end{abstract}

CoNCLUSIONS: In this model, arteries occluded by fibrin-rich clot demonstrated a significantly lower recanalization rate, lower final TIMI score, and a longer mean recanalization time than did arteries occluded by erythrocyte-rich clot. The angiographic outcome of MT by using the Merci clot retriever system was influenced by the histologic characteristics of the occluding thromboembolus.

ABBREVIATIONS: IMAX = internal maxillary artery; $\mathrm{LA}=$ lingual artery; $\mathrm{MT}=$ mechanical thrombectomy; SCA = superficial cervical artery; TIMI = thrombolysis in myocardial infarction; UCLA = University of California, Los Angeles

$\mathbf{M}_{\mathrm{T}}^{\mathrm{T}}$ $\mathrm{T}$ is an emerging treatment option for acute stroke. ${ }^{1-7}$ However, frequently 2 angiographically identical occluded vessels in patients with acute stroke will respond differently to MT for reasons that are not angiographically evident. One hypothesis is that the performance of the MT device is influenced by the histologic characteristics of thromboembolus (eg, fibrin/cellular component), but current image technology cannot differentiate thromboemboli of varying histologic characteristics. Given that the goal of MT is to optimize target-vessel recanalization and procedure time, it is crucial to

\section{Received March 30, 2011; accepted after revision June 23.}

From the Division of Interventional Neuroradiology (I.Y., I.K., R.H.K., A.G., F.A.V., F.V.), Department of Radiological Sciences, and Section of Neuropathology and Departments of Pathology and Laboratory Medicine and Neurology (H.V.V.), David Geffen School of Medicine, University of California, Los Angeles, Los Angeles, California; Department of Biostatistics (J.W.S.), UCLA School of Public Health, Los Angeles, Los Angeles, California; and Department of the Neurosurgery (Y.M.), Jikei University of Medicine, Tokyo, Japan. Harry V. Vinters is supported, in part by, the Daljit S. and Elaine Sarkaria Chair in Diagnostic Medicine and Specialized Programs of Translational Research in Acute Stroke, grant 1P50NS044378

Please address correspondence to Ichiro Yuki, MD, Division of Interventional Neuroradiology UCLA Medical Center and David Geffen School of Medicine at UCLA, 10833 Le Conte Ave, Los Angeles, CA 90095-1721; e-mail: iyuki@mednet.ucla.edu

http://dx.doi.org/10.3174/ajnr.A2842 know how the performance of an MT device is influenced by thromboemboli of differing histopathology.

A recent article reported that contrary to traditional teaching, approximately $75 \%$ of thromboemboli recovered from patients with stroke by MT showed complex histologic characteristics (a mixture of fibrin, erythrocytes, and nucleated cells). ${ }^{8}$ Moreover, the article showed that red clots composed solely of erythrocytes were uncommon.

We recently reported a new method for creating clot (fibrin-rich clot) with histologic characteristics similar to those of thromboemboli recovered from patients with stroke. ${ }^{9}$ This fibrin-rich clot is composed of histologically distinct layers: a fibrin-predominant layer, a cellular-predominant layer, and an erythrocyte-predominant layer. In contrast, the clot that is conventionally used in experimental thrombectomy models is homogeneous and predominantly composed of erythrocytes (erythrocyte-rich clot) with a meshwork of interspersed fibrin. The purpose of this study was to determine whether the efficacy of MT is influenced by thromboemboli having differing compositions. After embolization of the target vessels with clot of varying histology (fibrin- and erythrocyte-rich), we tested the performance of the Merci clot retriever device (Concentric Medical, Mountain View, California) on each clot type. 

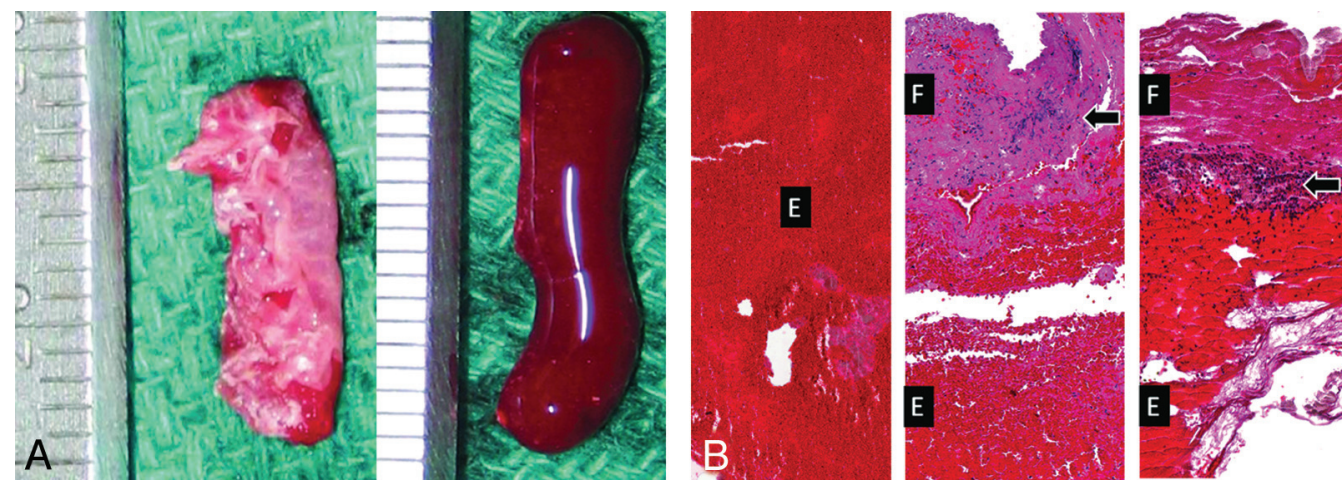

Fig 1. A, Macroscopic view of 2 different experimental clots. A novel experimental clot (fibrin-rich clot) (left) is prepared by manually excising the fibrin-rich layer of swine clot, which is created by natural sedimentation. Fibrin-rich clot shows more solid and elastic mechanical properties than conventional thrombin-induced experimental clot (erythrocyte-rich clot) (right). $B$, Microscopic view of the experimental clots versus thromboembolus recovered from a patient with acute stoke (H\&E, original magnification $\times 40$ ). A microscopic view of an erythrocyte-rich clot (left) demonstrates a thrombus composed predominantly of erythrocytes with interspersed fibrin and barium sulfate deposits. A thromboembolus recovered from a patient with stroke (center) shows distinct layers of fibrin, nucleated cells, and erythrocytes. A fibrin-rich clot (right) produces a layered pattern of fibrin, nucleated cells, and erythrocytes similar to that seen in the clot recovered from the patient with stroke (center). F indicates fibrin component; Arrows, the cellular component; $E$, the erythrocyte component.

\section{Materials and Methods}

\section{Animals}

All animal experiments were conducted in accordance with policies established by the UCLA chancellor's animal research committee and the National Institutes of Health guidelines. A total of 16 extracranial arteries in 5 Yorkshire swine (male and female animals; age range, 3-4 months; weight range, $30-40 \mathrm{~kg}$ ) were used in this study.

\section{Thrombus Preparation}

Preparation of Erythrocyte-Rich Clot. A conventional experimental clot (thrombin-induced clot) was prepared. The detailed preparation process is described elsewhere. ${ }^{10,11}$ Briefly, $10 \mathrm{~mL}$ of swine whole blood was mixed with $25 \mathrm{IU}$ of bovine thrombin (Dade Behring, Newark, Delaware). One-half a gram of barium sulfate was mixed with the blood for 10 seconds, and the mixture was kept in a silicone tube for 60 minutes at room temperature. After the incubation period, the created clot was removed from the silicone tube and cut to a length of $2 \mathrm{~cm}$ (Fig $1 A$ ).

Preparation of Fibrin-Rich Clot. The detailed preparation process of fibrin-rich clot is described elsewhere. ${ }^{9}$ Briefly, a syringe containing $1 \mathrm{~g}$ of barium sulfate powder was used to aspirate $30 \mathrm{~mL}$ of whole blood from an $8 \mathrm{~F}$ sheath placed in the right femoral artery. The syringe was then gently rotated to allow mixing of the whole blood and barium sulfate powder to obtain radiopacity. To minimize the anticoagulant effect of barium, which is due to absorption of vitamin $\mathrm{K}$-dependent coagulation factor $4,{ }^{12}$ we paid special attention to rotational motion during mixing. Subsequently, the contents of the syringe were incubated at room temperature, allowing separation of the whole blood components through precipitation, with plasma on top, fibrin/platelets/white blood cells in the middle, and red blood cells in the bottom. After plain sedimentation (2 hours), the solid component (fibrin-rich and erythrocyte-rich layers) was removed from the syringe. Next, the fibrin-rich layer was manually resected by using a number $11 \mathrm{scalpel}$, and pieces measuring $5 \mathrm{~mm}$ in diameter and $20 \mathrm{~mm}$ in length were prepared for injection (fibrin-rich clot) (Fig 1A).

Several samples of each clot type (fibrin- and erythrocyte-rich) were randomly selected and microscopically examined to evaluate their histologic characteristics (Fig 1B). The erythrocyte-rich samples were homogeneous in composition (composed almost exclusively of red blood cells) (Fig 1B, left). The fibrin-rich samples were complex in nature with well-defined fibrin-, cellular-, and erythrocyte-rich layers, respectively (Fig $1 B$, right).

Occlusion of Extracranial Arteries in Swine with Different Types of Experimental Clot. With the swine under general anesthesia, maintained with isoflurane $(2 \%-5 \%)$, an $8 \mathrm{~F}$ femoral sheath was placed in its right femoral artery. A high-resolution angiographic system (Allura Xper FD 10; Philips Healthcare, Best, the Netherlands) was used for selective digital subtraction angiography of each target artery. An $8 \mathrm{~F}$ balloon-guiding catheter (Concentric Medical) was advanced through the femoral sheath and placed at the origin of the target vessel. A control angiogram was obtained. The silicone tube containing a prepared clot was then connected to the guiding catheter, and approximately $5 \mathrm{~mL}$ of saline was injected to push the clot

\begin{tabular}{lccc}
\hline Comparison between the erythrocyte-rich clot group and the fibrin-rich clot group & \\
\hline & $\begin{array}{c}\text { Erythrocyte-Rich Clot } \\
\text { (Group A) }\end{array}$ & $\begin{array}{c}\text { Fibrin-Rich Clot } \\
\text { (Group B) }\end{array}$ & $\begin{array}{c}\text { Level of } \\
\text { Significance }\end{array}$ \\
\hline Total No. of treated vessels & 8 & 8 & N/A \\
Total No. of attempts (times) & 14 & 34 & $P<.001$ \\
Average No. of attempts per vessel (times) & 2.75 & 81.5 & $P<.001$ \\
Mean procedural time (min) & 15.5 & 4 & $P<.01$ \\
Final TIMl grade & & 1 & N/A \\
0 & 0 & 2 & 1 \\
2 & 0 & 3 & \\
3 & 5 & 3 & \\
4
\end{tabular}

Note:-N/A indicates not applicable. 


\section{Number of Attempts (clot retrieval)}
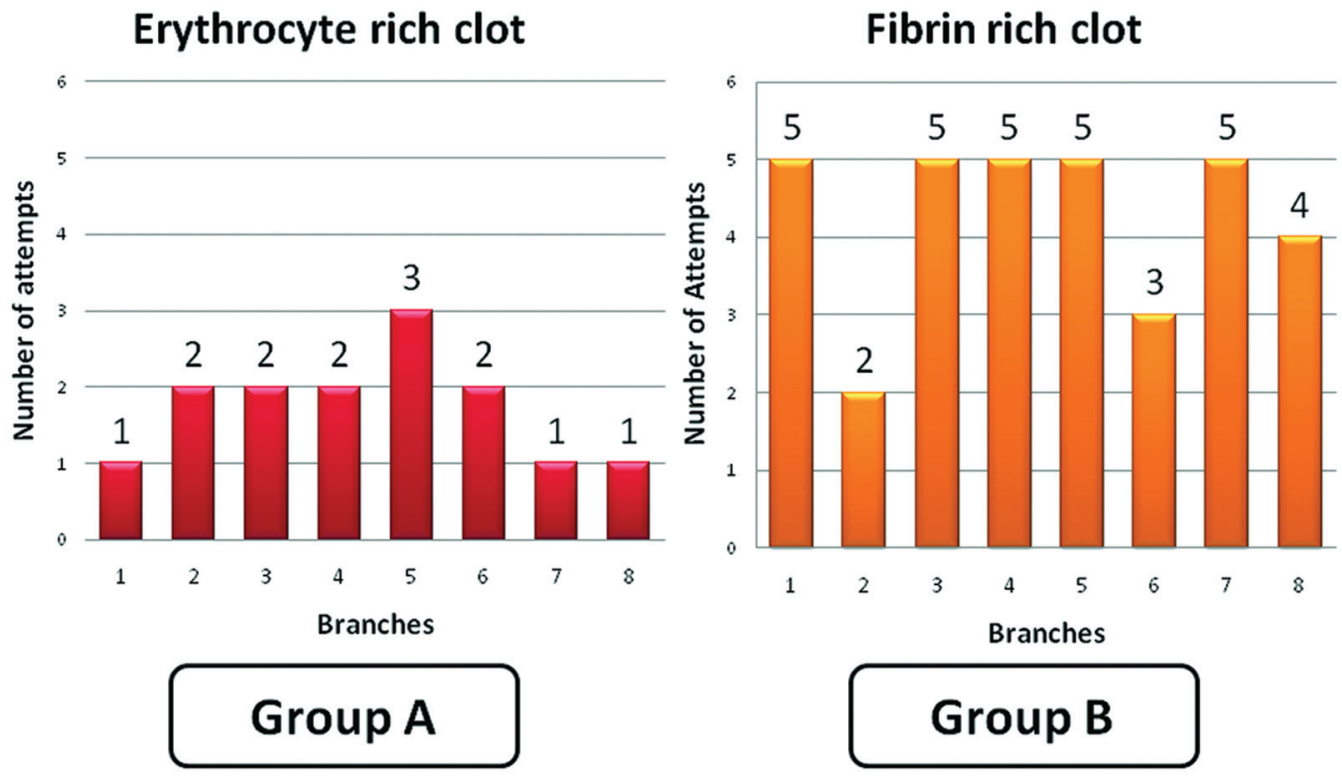

Fig 2. Bar graphs showing the number of attempts required to achieve the angiographic end point in each treated vessel. Every target vessel in group $A$ achieved recanalization of TIMI grade 2 or greater, whereas only 3 of 8 vessels achieved recanalization in group B. None of the treated vessels that underwent 5 attempts of retrieval demonstrated significant recanalization (1 vessel with TIMI 1 and 4 vessels with TIMI 0 ).

into the guiding catheter. Next, another $5 \mathrm{~mL}$ of saline was injected, under fluoroscopy, to inject the clot from the guiding catheter into the target artery. Target vessels included the LA, IMAX, and SCA. Vessels with complete occlusion were considered successfully embolized and used in the study. Vessels without complete occlusion after clot injection were excluded, and intervention was not performed. The target vessels occluded with conventional thrombin-induced (erythrocyterich) clot were categorized as group A, and the vessels occluded with fibrin-rich clot were categorized as group B.

\section{MT by Using Merci Clot Retriever System}

The use of extracranial vessels in swine to assess the efficacy of MT has been well established elsewhere. ${ }^{10,11,13}$ After the injection of experimental clot, the guiding catheter was removed for 5 minutes to restore arterial flow and allow embedding of the thrombus. The Merci clot retriever system was used for MT. The thromboembolus was crossed with a Transcend 14 guidewire (Boston Scientific, Natick, Massachusetts) loaded in an 18-L microcatheter (Concentric Medical), and the distal segment of the target vessel was then catheterized. A superselective angiogram was obtained via the microcatheter to confirm positioning of the catheter distal to the occlusion site. Depending on the size of the vessel, either a V2.5 or 3.0 (firm) thrombectomy device was selected. Next, a Merci clot retriever was deployed distal to the clot. The balloon catheter tip was inflated with $0.8 \mathrm{~mL}$ of $50 \%$ contrast solution. If the tip of the catheter was nearly-occlusive/occlusive at the origin of the vessel, the balloon was not inflated. Then, an attempt was made to pull the thrombus into the guiding catheter while gently aspirating. When the retriever system was immediately outside the guiding catheter, $30 \mathrm{~mL}$ of blood was vigorously aspirated while removing the Merci device. The balloon was then deflated, and both the Merci device and aspirate were examined for evidence of thrombus.
A postretrieval angiogram of the target artery was obtained and evaluated by using the TIMI scale. "Recanalization" was defined as TIMI 2 or greater in this study. The retrieval attempt could be repeated up to 5 times per target artery. If no recanalization was achieved after 5 attempts, the retrieval was rated as a failure. We collected the following data: 1) the number of attempts required to achieve recanalization, 2) distal migration or fragmentation of thrombus, 3) final angiographic result in the TIMI (0-3) scale, 4) the time (minutes) required to achieve the final result, and 5) vessel dissection or perforation. The presence of distal migration of thromboembolus and thrombus fragmentation was evaluated by comparing the preprocedural and postrecanalization angiograms. If the treated artery remained completely occluded in the postprocedural angiogram, the assessment was not performed.

\section{Statistical Data Analysis}

Each vessel tested in the study was considered to be an independent data point, and statistical analysis comparing the 2 groups (groups A and B) was performed. The Wilcoxon signed rank test (an unpaired nonparametric test) was used to compare the 2 groups in the following areas: 1) average number of attempts required to achieve the final angiographic result, 2) time (minutes) to achieve the final result, and 3) final TIMI grade.

\section{Results}

A total of 16 vessels (6 LAs, 8 SCAs, and 2 IMAXs) were occluded, and 48 retrieval attempts were made. A total of 8 vessels were successfully occluded with erythrocyte-rich clot (group A), and 8 vessels were occluded with fibrin-rich clot (group B). The Table summarizes the data and statistical significance between the 2 groups. In every target vessel, micro- 

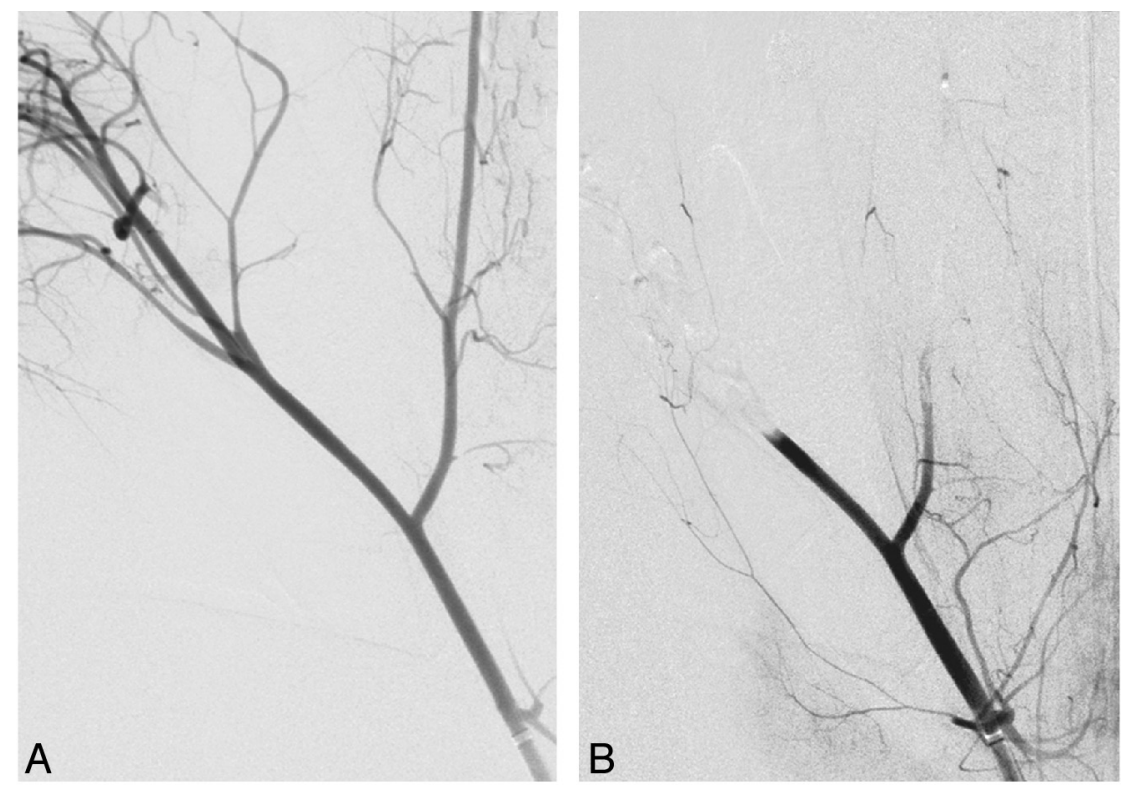

Fig 3. Angiographic findings of a target vessel occluded with an erythrocyte-rich clot and treated with MT. A, Preprocedural angiogram of a right superficial cervical artery (anteroposterior view) shows the branching pattern of the artery. $B_{\text {, }}$ Complete occlusion of the vessel was confirmed after injection of an erythrocyte-rich clot. C, A Merci clot retriever device (V2.5 firm) was deployed distal to the occlusion site, and clot retrieval was performed. D, A postprocedural angiogram shows recanalization of the treated artery. Small residual thrombi distal to the bifurcation of the artery are also seen (arrow).
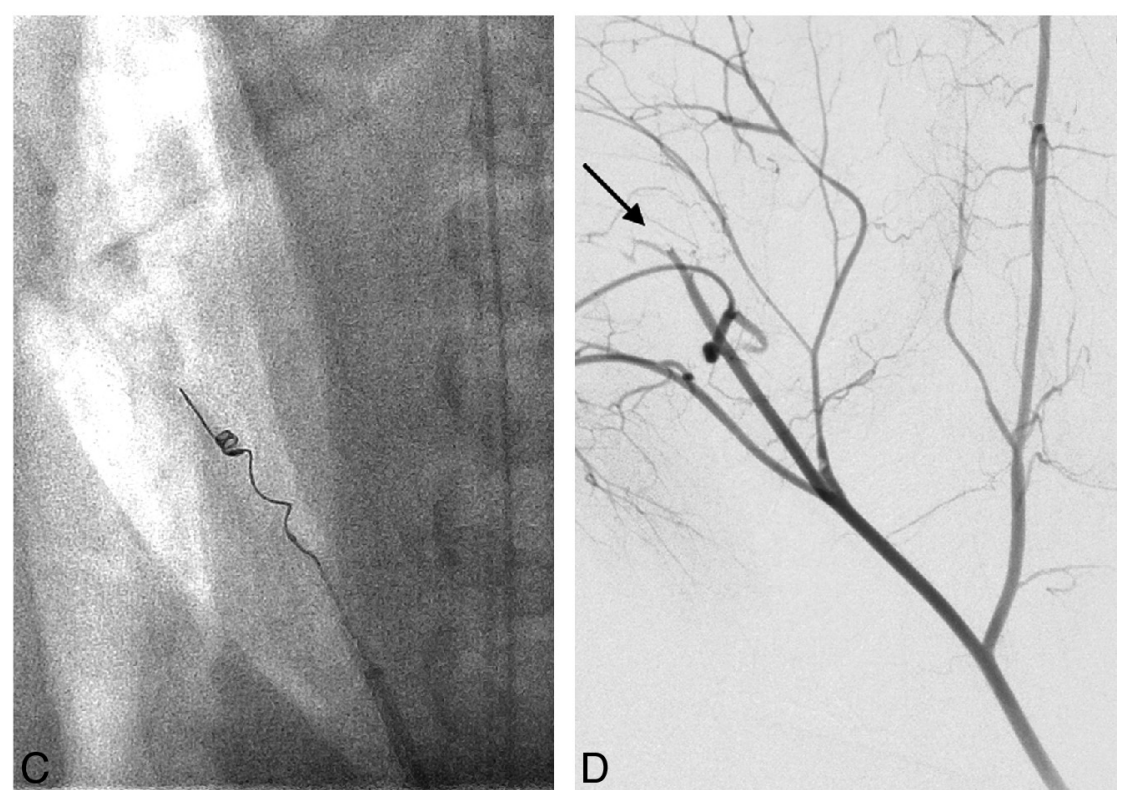

catheterization through the occlusion site was successfully achieved. Fourteen retrieval attempts were made to achieve the final angiographic results in the target arteries in group A, whereas 34 attempts were made in group $\mathrm{B}(P<.001)$. Every target vessel $(100 \%)$ in group A achieved recanalization of TIMI grade 2 or greater, whereas only 3 of 8 vessels (37.5\%) achieved recanalization of TIMI grade 2 or greater in group B. Figures 2 and 3 show the typical angiographic findings from each group. The average number of retrieval attempts to achieve the angiographic end point was 2.75 times in group $\mathrm{A}$ and 4.5 times in group $\mathrm{B}(P<.001)$. In group $\mathrm{A}, 3$ vessels demonstrated TIMI grade 3 recanalization with 1 retrieval attempt, 2 vessels showed TIMI grade 3 recanalization after 2 attempts, 2 vessels achieved TIMI grade 2 recanalization after 2 attempts, and 1 vessel showed TIMI grade 2 recanalization after 3 attempts. In group B, 1 vessel achieved TIMI 3 recanalization after 2 attempts, 1 vessel achieved TIMI 2 recanalization after 3 attempts, and 1 vessel achieved TIMI grade 2 recanalization after 4 attempts. The remaining 5 target vessels in group B did not demonstrate significant vessel recanalization ( 1 vessel with TIMI 1 after 5 attempts and 4 vessels with TIMI 0 after 5 attempts) (Fig 2).

The average time required to achieve the final angiographic result was 15.5 minutes in group $\mathrm{A}$ and 81.5 minutes in group B $(P<.01)$. Figures 3 and 4 show the typical angiographic findings from each group. All of the 8 target vessels in group A demonstrated recanalization (TIMI 2 or greater), and 7 of 8 demonstrated distal migration of the fragmented clot. In group B, only 3 of the 8 target vessels demonstrated recanalization, and 2 of 3 demonstrated distal migration of the fragmented clot. No vessel dissection or perforation was observed in the postprocedural angiograms.

We also observed the following trends: Erythrocyte-rich clots (group A) were more easily mobilized and demonstrated more fragmentation in comparison with fibrin-rich clots (group B). Complete stretching of the device at the site of occlusion was more frequently seen in group B. In group A, once the clot was engaged, it was typically successfully recov- 

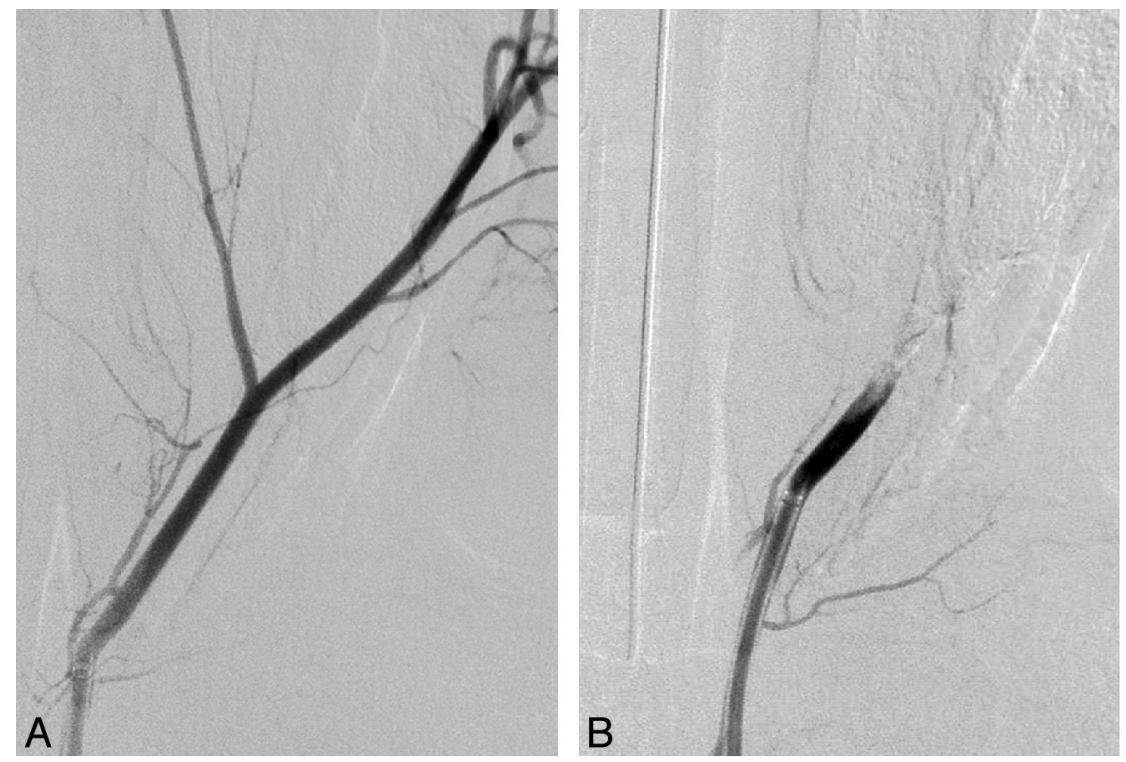

Fig 4. Angiographic findings of a target artery occluded with a fibrin-rich clot and treated with MT. A, A preprocedural angiogram of a left superficial cervical artery shows its branching pattern. $B$, Complete occlusion of the vessel was confirmed after injecting a fibrin-rich clot. C. A Merci clot retriever device (V2.5 firm) was deployed distal to the clot, and clot retrieval was performed. D, After 5 attempts at clot retrieval, the postprocedural angiogram shows that the treated vessel remains occluded.
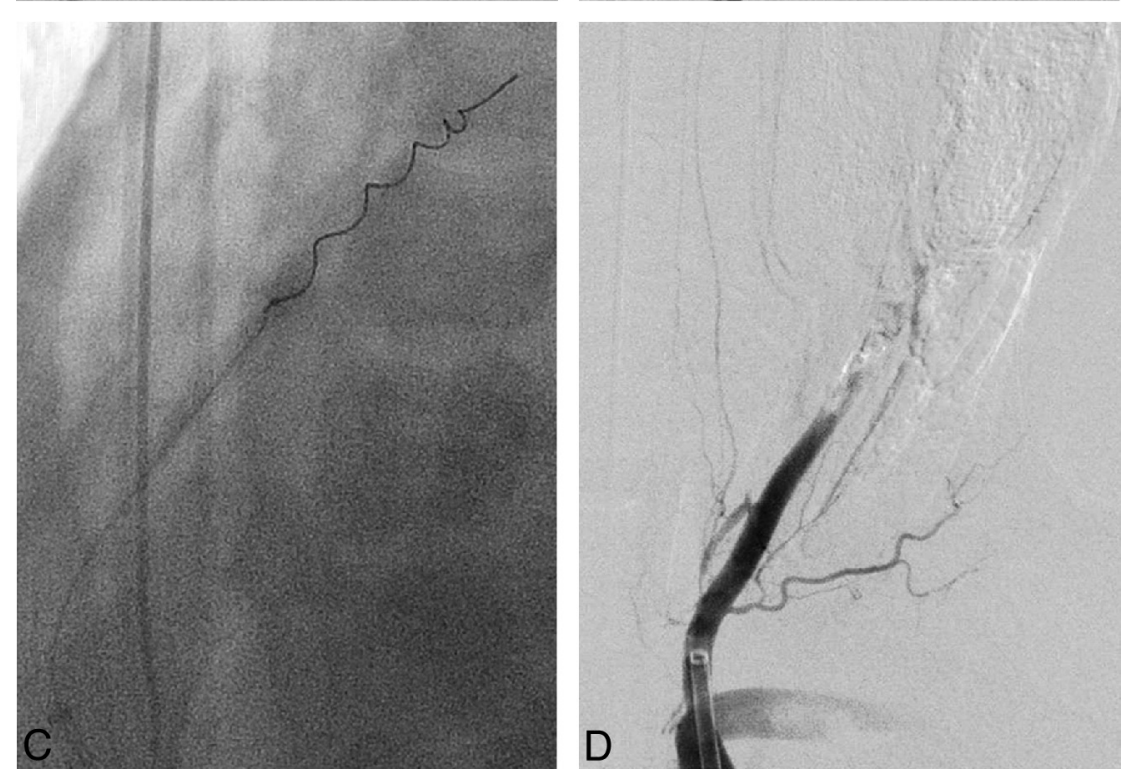

ered, whereas in group B recovery was typically unsuccessful after clot capture.

\section{Discussion}

\section{Histologic Characteristics of Thromboemboli Recovered from Patients with Stroke}

Marder et $\mathrm{al}^{8}$ performed histologic analysis on 25 thromboemboli from 25 separate patients with acute stroke, recovered with MT. The study showed that a majority $(75 \%)$ of the recovered thromboemboli were fibrin-rich and that so called "red clots" composed uniquely of erythrocytes were uncommon. In this study, we created 2 histologically distinct types of clot: 1 with fibrin/cellular/erythrocyte components, which mimic clot recovered from patients with acute stroke, and 1 composed predominantly of erythrocytes.

\section{Thrombectomy Device Performance Can Be Influenced by} the Histologic Characteristics of Thromboemboli

In our experience, target vessels occluded by fibrin-rich thromboemboli required more attempts at thrombectomy to achieve recanalization (TIMI grade 2 or 3 ) compared with vessels occluded by erythrocyte-rich clot ( 4.5 times versus 2.8 times respectively, $P<.001$ ). Consequently, the mean time to the angiographic end point in the fibrin-rich group was longer than that in the erythrocyte-rich group (81.5 minutes versus 15.5 minutes, $P<.01$ ). Furthermore, every vessel occluded with erythrocyte-rich clot showed recanalization defined as a final TIMI grade of 2 or 3 (100\%), whereas only 3 of $8(37.5 \%)$ vessels occluded with fibrin-rich clot achieved recanalization.

These results suggest that vessels occluded with fibrin-rich clot are relatively more resistant to MT than those occluded with erythrocyte-rich clot. There are a few interpretations as to why recovering fibrin-rich clot is more challenging: 1) One is that the fibrin and cellular components of fibrin-rich clot make it stiff/hard. The stiff nature of the fibrin-rich clot may reduce the probability of successful engagement by the MT device, in turn decreasing the chances of successful clot capture. The stiff/noncompliant nature of fibrin-rich clot may also explain why complete stretching of the device at the occlusion site is more frequently observed in group B. 2) It is also 
possible that erythrocyte-rich clot fragments easily make piecemeal clot retrieval feasible, whereas fibrin-rich clot is less likely to fragment, preventing piecemeal thrombectomy, due to its more rigid nature. This partially explains why embolization in distal branches is frequently seen in the erythrocyterich clot group. 3) Last, on more than 1 occasion, a fibrin-rich clot was successfully captured by the MT device, but the captured clot would not pass through the guiding catheter tip. The explanation, while speculative on our part, may be secondary to the mechanical properties of fibrin-rich clot (ie, the noncompliant nature of the fibrin-rich-clot and its tendency to disengage from the MT device at the tip of the guiding catheter).

\section{Limitations of the Study}

The main limitation was the small sample size of our study. The use of only 1 MT device, the Merci clot retriever, which was necessary given our limited sample size, was another limitation. Finally the use of only 1 clot size $(2.0 \times 0.5 \mathrm{~cm})$ was also a limitation. In an animal experiment, Gralla et $\mathrm{al}^{14}$ reported that occlusion length is highly associated with the success rate in MT. Additional investigation with larger numbers of subject animals is necessary to further test many of the hypotheses we have set forth.

The variable radiopacity of the fibrin-rich clot was a technical limitation in this study. To minimize the anticoagulant effect of barium (explained by absorption of vitamin $\mathrm{K}$-dependent coagulation factors), ${ }^{12}$ we carefully controlled the mixture of barium and whole blood. Given the limited mixture time of barium and whole blood, there was heterogeneity in the radiopacity of the fibrin-rich clot samples. Therefore, developing a technique that consistently produces fibrin-rich clot with homogeneous radiopacity is requisite.

\section{Other Potential Factors That Influence the Performance of MT Devices}

There are other factors that potentially impact the performance of the MT devices. Yin et al $^{15}$ described 5 postmortem cases of patients with acute stroke who underwent MT with unsuccessful recanalization. Four of 5 patients showed prominent atheromata, often with significant luminal stenosis in the treated lesion. Although limited by a small sample size, the study by Yin et al presents another potential explanation for resistance to MT (baseline vessel stenosis).

\section{Future Applications of a Stroke Model by Using Fibrin-Rich Clot}

It is critical to evaluate the devices under challenging and reproducible conditions, which is best done by using an animal model, to make improvements on existing and experimental MT devices. Given that the mechanical and histologic features of a thromboembolus are potentially among the key variables in predicting MT device performance, the use of a model with conventional thrombin-induced as well as fibrin-rich clot may contribute to the further refinement of MT devices.

Furthermore, future improvements in diagnostic imaging technology may allow histologic characterization of in situ clot. This would make information from an animal model (ie, MT device performance as a function of clot histology) clinically applicable and could potentially play a role in procedure planning.

\section{Conclusions}

To evaluate whether the efficacy of mechanical thrombectomy is influenced by the histologic characteristics of thromboemboli, swine vessels occluded by 2 different types of experimental clot were treated with a MT device, and the angiographic outcomes were analyzed. Target vessels occluded by fibrinrich clot demonstrated significantly lower recanalization rates, longer mean recanalization time, and lower final TIMI scores compared with the vessels occluded by erythrocyte-rich clot. In our study, the performance of the Merci clot retriever system was influenced by the histologic characteristics of the thromboemboli.

Disclosures: Harry V. Vinters_UNRELATED: Stock/Stock Options: have significant share holdings in Minnesota Mining and Manufacturing Company, Glaxo-Smith-Kline, Teva Pharma, and Pfizer. I received dividends from all these companies. Fernando A. VinuelaRELATED: Provision of Writing Assistance, Medicines, Equipment, or Administrative Support. Volunteer.

\section{References}

1. Gobin YP, Starkman S, Duckwiler GR, et al. MERCI 1: a phase 1 study of Mechanical Embolus Removal in Cerebral Ischemia. Stroke 2004;35:2848-54

2. Smith WS, Sung G, Saver J, et al. Mechanical thrombectomy for acute ischemic stroke: final results of the Multi MERCI trial. Stroke 2008;39:1205-12

3. Bose A, Henkes H, Alfke K, et al. The Penumbra System: a mechanical device for the treatment of acute stroke due to thromboembolism. AJNR Am J Neuroradiol 2008;29:1409-13

4. Castano C, Dorado L, Guerrero C, et al. Mechanical thrombectomy with the Solitaire $\mathrm{AB}$ device in large artery occlusions of the anterior circulation: a pilot study. Stroke 41:1836-40

5. Josephson SA, Saver JL, Smith WS. Comparison of mechanical embolectomy and intraarterial thrombolysis in acute ischemic stroke within the MCA: MERCI and Multi MERCI compared to PROACT II. Neurocrit Care 2009;10: 43-49

6. Nogueira RG, Schwamm LH, Hirsch JA. Endovascular approaches to acute stroke. Part 1. Drugs, devices, and data. AJNR Am J Neuroradiol 2009;30: 649-61

7. Nogueira RG, Yoo AJ, Buonanno FS, et al. Endovascular approaches to acute stroke. Part 2. A comprehensive review of studies and trials. AJNR Am J Neuroradiol 2009;30:859-75

8. Marder VJ, Chute DJ, Starkman S, et al. Analysis of thrombi retrieved from cerebral arteries of patients with acute ischemic stroke. Stroke 2006;37: 2086-93

9. Kan I, Yuki I, Murayama Y, et al. A novel method of thrombus preparation for use in a swine model for evaluation of thrombectomy devices. AJNR Am J Neuroradiol 2010;31:1741-43. Epub 2010 Jan 21

10. Gralla J, Schroth G, Remonda L, et al. A dedicated animal model for mechanical thrombectomy in acute stroke. AJNR Am J Neuroradiol 2006;27:1357-61

11. Gralla J, Schroth G, Remonda L, et al. Mechanical thrombectomy for acute ischemic stroke: thrombus-device interaction, efficiency, and complications in vivo. Stroke 2006;37:3019-24

12. Zucker MB, Owen J. Non-decalcified barium sulfate-adsorbed plasma: a potentially useful reagent for studying blood clotting, platelets or complement. Thromb Haemost 1982;47:182-84

13. Brekenfeld C, Schroth G, El-Koussy M, et al. Mechanical thromboembolectomy for acute ischemic stroke: comparison of the catch thrombectomy device and the Merci retriever in vivo. Stroke 2008;39:1213-19

14. Gralla J, Burkhardt M, Schroth G, et al. Occlusion length is a crucial determinant of efficiency and complication rate in thrombectomy for acute ischemic stroke. AJNR Am J Neuroradiol 2008;29:247-52

15. Yin NS, Benavides S, Starkman S, et al. Autopsy findings after intracranial thrombectomy for acute ischemic stroke: a clinicopathologic study of 5 patients. Stroke 2010;41:938-47 